\title{
The Impact of Well - Being Therapy on Symptoms of Depression
}

\author{
Majid Moeenizadeh \\ Faculty of Education and Psychology, Ferdowsi University of Mashhad, Iran \\ E-mail: moinyzadeh@gmail.com \\ Kiran Kumar K. Salagame \\ Department of Studies in Psychology, University of Mysore, Mysore 570006, India \\ E-mail: kiku@psychology.uni-mysore.ac.in
}

\begin{abstract}
Well Being Therapy (WBT) is a relatively modern technique of managing patients with Depression. Many studies and researches have been conducted in this field to formally found the Positive Psychology, the goal of which is to study the nature of wellbeing and the conditions, which stimulate them. The improvement shown by patients who were managed with this technique lead to WBT based on Ryff's multidimensional model of psychological well-being. This article has been written to emphasize the novel applications of Psychotherapeutic Strategy for patients suffering from depression. The objective of this therapy is to improve the level of psychological well-being of patients. WBT has been executed in 10 sessions according to Ryff's phases. Methods: A sample of 40 patients was treated in 8session by WBT and CBT and assessed (BDI-II) for specification of depression as pre and post tests. Results: Test-retest of WBT and CBT showed a more significant effect between pre and post treatment and WBT is more effective than CBT. Conclusions: The results suggested the feasibility and clinical advantages by adding WBT to the treatment of depression.
\end{abstract}

Keywords: Positive Psychology, Well-Being, Well-Being Therapy (WBT), Beck Depression Inventory (BDI-II) and Depression.

\section{Introduction}

Depression is one of the most common psychiatric disorders. The causal factors could range from genetic to socio-economic conditions prevalent in the society in which the patient lives. Prevalence of depressive disorders varies in different socio-cultural populations. In a study on depressive disorders in 8764 randomly selected samples of general population in five European countries, the prevalence was assessed by a cross-sectional two-phase community study using Beck's Depression inventory in Phase 1 and Schedule for Clinical Assessment in Neuropsychiatry in Phase 2. The Overall prevalence of depression was $8.56 \%$. The weighted prevalence of different diagnostic categories of depressive disorders was as follows; major depressive episode: 6.7\%, dysthymia: $1 \%$ and adjustment disorder: $0.5 \%$ (Ayuso-Mateos, et al., 2001).

In a cross-sectional population-based epidemiological study in Iran, 25180 individuals who aged more than 18 were assessed using Schedule for Affective Disorders and Schizophrenia (SADS). The current prevalence of depressive disorders was $2 \%$ (1.8\% major depressive disorder and $0.2 \%$ minor depressive disorder), and it was associated with female gender, lower education, being married, being middle-aged, living in cities and not being a homemaker (Mohammadi, et al., 2006).

Noorbala et al (1987) studied 879 individuals to estimate the prevalence of psychiatric disorders in Tehran (the capital of Iran). Using DSM IV criteria and clinical interview, they concluded that the prevalence of depressive disorders was $9.2 \%$ (4.4\% major depressive disorders, $3.9 \%$ dysthymia and $0.8 \%$ minor depressive disorder) (Noorbala, Mohammadi, 1987).

Indeed, depression usually causes substantial imperfection of both occupational and social functioning. It is classified among the most costly and weakening of illnesses worldwide (Keller \& Boland, 1998). In recent years, an increased public recognition of the devastating personal and financial implications of depression has lead to identifying effective and preventative psychological interventions for this disorder, and Aaron Beck's (1967) cognitive-behavioral therapy (CBT) is the most researched of these. The two major treatment goals of CBT are (a) teaching patients to modify their dysfunctional thoughts as a means of ameliorating depressive symptoms; and (b) giving patients with a set of enduring cognitive skills to reduce the risk of subsequent relapse. 
An altogether different approach to the treatment of depression is to enhance the sense of well-being in the patient, instead of reducing the symptoms. This approach is rooted in the contemporary developments in the study of well-being leading to the emergence of Positive Psychology. One method of treatment that is being tested on a variety of depressive patients is Well-Being Therapy (WBT) developed by an Italian psychiatrist Giovanni Fava ( Fava, Rafanelli, Grandi, Conti, \& Belluardo, 1998; Fava, Rafanelli, Cazzaro, Conti, \& Grandi, 1998a; . Fava, 1999; Fava, \& Mangelli., 2001; Fava \& Ruini, 2003; Fava, Ruini, Rafanelli, Finos, Salmaso, Mangelli., 2005). This technique appears to be more effective as compared to the standard CBT technique (Moeenizadeh and Salagame, in press).

\section{Background of Well- Being Therapy}

Psychological well-being (WB) is viewed as not only the absence of mental disorder but also the presence of positive psychological resources, including components of hedonic or subjective well-being (e.g., positive affect, life satisfaction, happiness - Diener, 1984), as well as components of eudemonic well-being (e.g., self-acceptance, positive relations, autonomy, purpose in life- Sin \& Lyubomirsky, 2009). The approaches to increase well-being are generically termed as "positive psychology interventions (PPIs)", which refer to "treatment methods or intentional activities that aim to cultivate positive feelings, behaviors, or cognitions". As practitioners advice PPIs can be used in the treatment of both clinically depressed and non-depressed clients. Fava points out that PPIs are especially effective for treating residual symptoms (Fava, et al., 1998) and avert future relapse for formerly depressed clients (Seligman, Rashid, \& Parks, 2006).

The development of well-being therapy originated from three converging developments. First, there is a growing body of literature on residual symptoms after apparently successful treatment in mood and anxiety disorders. Second, the partial remission after treatment was not found to be limited to negative affective symptoms. Finally, clinicians working with patients with mood and anxiety disorders are often confronted with the unsatisfactory degree of remission that current therapeutic strategies yield and with the vexing problems of relapse and recurrence ( Fava, Tomba, \& Grandi, 2007).

A question that may arise is what differentiates well-being therapy from standard cognitive therapies, which may also involve positive thinking. A main difference between standard cognitive therapies and well-being therapy is thus the focus, which in well-being therapy is on instances of emotional well-being, whereas in cognitive therapy it is on psychological distress. The second important distinction is that in cognitive therapy, the goal is abatement of distress through automatic thought control, whereas in well-being therapy the goal is a promotion of psychological well-being along Ryff's (1989) dimensions. The final distinction is that, unlike cognitive behavioral frameworks, well-being therapy refrains from explaining from the outset, to the patient its rationale and strategies but relies on his or her progressive appraisals of positive self. Enhancing acceptance (vs. control) of distress and positive resources to keep distress in bounds and fostering individualized road to recovery, set by the patient's increasing awareness, is another characteristic of well-being therapy (Giovanni, Fava, \& Tomba, 2009).

Well-being therapy is based on an educational model which is structured, directive, and oriented to present problems and situation ( Fava, 1999; Fava \& Ruini, 2003). It is a short-term psychotherapeutic strategy that can be implemented over 8-12 sessions, every week or alternate weeks. Well-Being Therapy involves 'technique of self observation' along with the 'use of a structured diary' and 'interaction between patients and therapist'. For details of the therapy, one may refer to Fava and Tomba, (2009).

\section{Objectives}

The aim of this study was to apply a novel psychotherapeutic approach to increase the level of well-being in persons suffering from depression. The objective of this study was to examine the efficacy of CBT and WBT, in terms of enhancement of well-being and reduction of symptoms. The findings related to the enhancement of well-being is reported elsewhere (Moeenizadeh and Salagame, in press). Here the findings related to the reduction of symptoms as measured through Beck's Depression Inventory are reported. This study can provide psychologists, new ways of using a psychotherapeutic approach. Since the study is first author's doctoral work, only the findings related to Beck's Depression Inventory is reported here.

\section{Methodology}

\subsection{Design}

The study employed pre-test and post-test design to find the differences between CBT and WBT groups. The independent variable was the therapeutic aspects of WBT and CBT, and the dependent variable was the condition of patients suffering from depression. 


\subsection{Sample}

The statistical universe of this research was adolescent and adult students suffering from depression who visited Ferdowsi University Clinic and other affiliated clinics, in Iran. Forty male and female patients suffering from major depressive disorders as per DSM IVR criteria were chosen for the study, using Krejcie and Morgan, (1970) criteria. The subjects (20 male and 20 female) were randomly assigned to either WBT or CBT. Attempt was made to match the two groups based on demographic factors like gender, age, educational level and socio-economic status.

\subsection{Tools}

Three tests were used before starting the treatment. The first and second session were spent in administrating Persian version of Beck Depression Inventory (BDI-II) (Ghassemzadeh, Mojtabai, Karamghadiri, \& Ebra-himkhani 2005), Psychological Well-being Scale (PWB) (Ryff, 1989), and Rorschach Inkblot Test (Rorschach, 1921). The subjects were reassessed with the BDI-II, PWB and Rorschach after treatment (8 sessions) by the same researcher who had performed the previous evaluations and who was blind to the treatment assignment.

Beck, Steer and Brown revised The BDI in 1996, and is known as BDI-II. The BDI-II is a 21-item self-report instrument with items describing the cognitive-affective factor and somatic-vegetative factor of depression. Dozois et al. [1998] proposed two-factor solution in a much larger sample of Canadian college students, and similar to Beck and colleagues [1996]. BDI-II is divided in two dimensions: (a) Items 1 to 3, 5 to 9 , and 13 to 14 loaded on the cognitive-affective factor, and (b) Items 4, 10 to 12, and 15 to 21 loaded on the somatic-vegetative factor. The items related to cognitive affective factors are sadness, pessimism, past failure, guilty feeling, punishment feeling, self-dislike, self-criticalness, suicidal thoughts, indecisiveness, and worthlessness. The items related to somatic-vegetative factors are loss of pleasure, crying, agitation, loss of interest, loss of energy, changing in sleep, irritability, change in appetite, concentration difficulty, tiredness or fatigue, and loss of interest in sex Items are arranged according to the severity of the content of alternative statements and are rated on a four-point scale. BDI-II has been translated into Persian and is validated by Ghassemzadeh, Mojtabai, Karamghadiri, \& Ebra-himkhani (2005). The BDI-II-Persian version has a high internal consistency (Cronbach's $\alpha=0.87)$ and acceptable test-retest reliability $(r=0.74)$. This version was used in the present study.

\subsection{Procedure}

The data in our research were collected between June 2008 and October 2008. The Before administering the questionnaires and treatment the nature of the study and research objectives were, explained to them.

Eight sessions of WBT were conducted. The duration of each session was 45 to 60 minutes. The therapy was conducted over a period of eight weeks. WBT involved technique of self-observation along with the use of a structured diary and interaction between patients and therapists. The therapy sessions were divided into three phases - Initial, Intermediate and Final. The first two sessions (the initial phase), were simply concerned with identifying and setting episodes of well-being into situational context, no matter how short-lived they were (Fava, 1999). Patients were requested to maintain a report in the form of a structured diary, the circumstances surrounding the episodes of well-being, rated on a scale of 0 to 100 , with 0 being an absence of well-being and 100 the most intense well-being.. In the 3rd to 5th session (the intermediate phase), the patient was encouraged to identify thoughts and beliefs leading to premature interruption of well-being. In the 6th to 8th sessions (final phase), patients were assessed according to Ryff's dimensions of well-being such as Environmental Mastery, Personal Growth, Purpose in Life, Autonomy, Self-Acceptance and Positive Relations with others (Ryff \& Singer, 1996). Therefore, the goal of the therapist was to guide the patient from an impaired level to an optimal level according to the above six dimensions.

Another group of clients was treated with standard CBT techniques developed by Arnold Beck. At the end of the sessions, post-assessment was conducted for each client using Beck Depression Inventory (BDI-II).

\section{Results and Discussion}

The obtained data were analyzed using Repeated Measures ANOVA. On the measure of depression, there are significant differences between pre scores (Mean of pre $\mathrm{CBT}=33.00$ ) and post scores (Mean of post $\mathrm{CBT}=12.35$ ). This result was repeated in WBT group (Mean of pre WBT=45.35 and Mean of post $\mathrm{CBT}=12.35$ ). As Table 1 shows, there is a significant difference between pre and post scores.

Table1: Pre and post-scores on BDI II for CBT and WBT Groups 


\section{Table2: Effect size for the scores of CBT and WBT groups}

For more clarity, Chart1 also illustrates the results:

The findings show that all the patients who underwent WBT and CBT gained significantly. However, as the effect sizes (Table 2) show patients who were treated with WBT showed greater symptom reduction than the other group. This demonstrates that WBT not only enhances the sense of well-being but also can significantly decrease the symptoms. Thus, findings of the study are expected to be beneficial in planning more effective treatment programs for treating patients with depression.

The obtained data were also analyzed using Repeated Measures ANOVA for BDI-II subscales (see Table 3). The results demonstrated that there are significant differences between pre and post subscales scores of BDI-II inventory in CBT group. In addition, in WBT group, only the significant subscales are mentioned in the Table.

\section{Table 3: F and $p$ values for the sub-scale scores of BDI II for CBT and WBT groups}

As mentioned above scores on 10 items of 21BDI-II, were significant in CBT and WBT; these significant items consisted of both cognitive affective and somatic factors, six items in cognitive affective and four items in somatic factor. It means although WBT mean score was more than CBT in pre-test it changed and the improvement was significantly more than CBT in post-test. Therefore, it demonstrates that WBT has more effectiveness than CBT.

To affirm this significance correlation between post BDI-II and post PWB scores was also calculated. The results showed a significant negative relationship (See table4 and chart2).

\section{Table4:}

The results also illustrated by Chart3.

\section{Chart2:}

According to this significant negative relationship, clearly when well-being increase depression decreases. It indicates that well-being increased after treatment by WBT. The above results of this investigation lend support to previous literature reviewed in detail elsewhere (Ruini \& Fava, 2009). It also supports the results of other findings (Fava \& Ruini, 2003). The present study showed that all patients who took part in (8 sessions) of WBT group improved more and have close relationships, optimistic, happiness, innocence, success, and socialization, with others, than the patients participated in ( 8 sessions) of CBT groups. Therefore, it seems and suggests that treating with WBT could be more effective than treating with CBT.

\section{Discussion}

The purpose of this study was to apply a new method of therapeutic intervention for depression - Well-Being Therapy (WBT) and compare it to CBT. The findings of this study show that all the patients who underwent WBT and CBT gained significantly in terms of symptom reduction as measured by BDI_II. The patients who were treated with WBT have shown a significant decrease in depression indicating the efficacy of this novel technique. As the effect sizes show patients, who were treated with WBT showed greater symptom reduction than the other group.

In the present study ten of twenty-one symptoms sadness, pessimism, past failure, guilty feeling, indecisiveness, and worthlessness (related to cognitive-affective factor) and crying, agitation, loss of interest, and loss of interest in sex (related to somatic-vegetative factor) showed the significant changes. This demonstrates that WBT not only decrease cognitive-affective factor but also can significantly decrease somatic-vegetative factor.

At this stage, the causal mechanisms involved in the effectiveness of WBT in decreasing symptoms of depression is still not clear. As Wood and Joseph (2010, p. 214) point out it is not clear whether the absence of positive well-being can predict the clinically relevant levels of depression, or simply predict variance in sub-clinical melancholia. Nevertheless, from their recent study on 5778 persons who had participated in Wisconsin Longitudinal Cohort Survey, which began in 1957, they found that people with low positive well-being were 7.16 times more likely to be depressed 10 years later. They also found that after controlling for personality, negative functioning, and prior depression, demographic, economic, and physical heath variables, people with low positive well-being were still over twice as likely to be depressed. They concluded that the results of their study suggest that in addition to having negative characteristics, the absence of positive characteristics forms an additional (and substantial) risk factor for depression. Hence, it is likely that the alleviation of low PWB can be a preventative measure for depression and alleviating low PWB in psychiatric practice could potentially form an innovative new cure for depression. 
As evident from the findings of this study reported separately (Mooenizadeh \& Salagame, in press) all, the patients who underwent WBT and CBT gained significantly in PWB scores also. The gain was more in WBT group as compared to CBT group. In four of the six dimensions of PWB, the patients who were treated with WBT showed a significant increase in psychological well-being indicating the efficacy of this novel technique. They are autonomy, environmental mastery, purpose in life, and self-acceptance. As Maddux (2004) states, the intervention of positive psychology lays stress on the improvement of strengths and assets of the patients. It is guided by the belief that strengthening the strengths will weaken the weaknesses. Perhaps this explains the reduction in the symptoms of depression as found in this study.

\section{Limitations}

Perhaps the greatest limitation of this study relates to following up of the patients. Follow up could not be conducted because some of the students were studying in a last semester and after completing their studies returned to their city, and they could not be traced easily. Additionally, some of them declined to give any information about their place of residence. Further, since the researcher had to return to India where he had enrolled for Ph.D, contacting the patients was difficult. Another limitation is that the sample in this study consisted of only students, and hence the findings cannot be generalized to another population. Moreover, the sample of this study was limited to only those suffering from dysthymic depressive disorder, and we do not know how patients suffering from other kinds of depression disorders will respond to WBT.

\section{Conclusion}

WBT is based on inferences arrived at, from the Positive Psychology Literature. The objective of WBT is to achieve the optimal level of well - being by helping them to realize their true potential. As is evident, WBT is still at evolving stage. The article has employed well-being therapy to examine the effectiveness of WBT for treatment of patients suffering from depression. The result has shown a considerable reduction in depression disorder.

\section{Reference}

Ayuso-Mateos JL, Vazquez-Barquero JL, Dowrick C, Lehtinen V, Dalgard OS, \& Casey P, et al. (2001). Depressive disorders in Europe: Prevalence figures from The ODIN study. British Journal of psychiatry, 179, 308-316.

Beck, A. T. (1967). Depression: Clinical, experimental, and theoretical aspects. New York: Harper \& Row.

Beck, A. T., Steer, R. A., Ball, R., \& Ranieri, W. (1996). Comparison of Beck Depression Inventories -IA and -II in psychiatric outpatients. J Pers Assess, 67(3), 588-597.

Beck, A. T., Ward, C. H., Mendelson, M., Mock, J., \& Erbaugh, J. (1961). An inventory for measuring depression. Arch Gen Psychiatry, 4, 561-571.

Cohen, J. (1988). Statistical power analysis for the behavioral sciences (2nd ed.). Hillsdale, NJ: Erlbaum.

Diener, E. (1984). Subjective well-being. Psychological Bulletin, 95, 542-575.

Dozois, D. J., Dobson, K. S., \& Ahnberg, J. L. (1998). A psychometric evaluation of the Beck Depression Inventory-II. Psychol. Assess, 10, 83-89.

Fava, G. A. (1999). Well-being therapy: conceptual and technical issues. Psychother Psychosom, 68(4), 171-179.

Fava, G. A., \& Mangelli, L. (2001). Assessment of subclinical symptoms and psychological well-being in depression. Eur Arch Psychiatry Clin Neurosci, 251 Suppl 2, II47-52.

Fava, G. A., \& Ruini, C. (2003). Development and characteristics of a well-being enhancing psychotherapeutic strategy: well-being therapy. J Behav Ther Exp Psychiatry, 34(1), 45-63.

Fava, G. A., \& Sonino, N. (2000). Psychosomatic medicine: emerging trends and perspectives. Psychother Psychosom, 69(4), 184-197.

Fava, G. A., \& Tomba, E. (2009). Increasing Psychological Well-Being and Resilience by Psychotherapeutic Methods. J Pers.

Fava, G. A., Rafanelli, C., Cazzaro, M., Conti, S., \& Grandi, S. (1998a). Well-being therapy. Psychol. Med, 28, 475-480.

Fava, G. A., Rafanelli, C., Grandi, S., Conti, S., \& Belluardo, P. (1998). Prevention of recurrent depression with cognitive behavioral therapy: preliminary findings. Arch Gen Psychiatry, 55(9), 816-820.

Fava, G. A., Ruini, C., Rafanelli, C., Finos, L., Salmaso, L., Mangelli, L., et al. (2005). Well-being therapy of 
generalized anxiety disorder. Psychother Psychosom, 74(1), 26-30.

Fava, G. A., Tomba, E., \& Grandi, S. (2007). The road to recovery from depression. Psychotherapy and Psychosomatics, 76, 260-265.

Garber, J., \& Flynn, C. (2001). Vulnerability to depression in childhood and adolescence In R.E.Ingram \& J.M.Price, (Eds). Vulnerability to psychopathology: risk Across the Lifespan (pp. 175-293.) New York: Guilford Press.

Ghassemzadeh, H., Mojtabai, R., Karamghadiri, N., \& Ebrahimkhani, N. (2005) psychometric properties of a persian language version of the Beck Depression Inventory (2 ed). BDI-II persian. Depression and anxiety, (pp.1-7). Wiley-Liss, Inc.

Giovanni, A., Fava, G. A., \& Tomba, E. (2009). Increasing psychological well-being and resilience by psychotherapeutic methods. Journal of Personality, 77.

Keller, M. B., \& Boland, R. J. (1998). Implications of failing to achieve successful long-term maintenance treatment of recurrent unipolar major depression. Biol Psychiatry, 44(5), 348-360.

Kessler, R. C., McGonagle, K. A., Nelson, C. B., Hughes, M., Swartz, M., \& Blazer, D. G. (1994). Sex and depression in the National Comorbidity Survey. II: Cohort effects. $J$ Affect Disord, 30(1), 15-26.

Krejcie, R. V., \& Morgan, D. W. (1970). Determining Sample Size for Research Activities. Educational and Psychological Measurement, 30, 607-610.

Maddux, S., \& Lopez, S. J. (2004). Toward a positive clinical psychology: Deconstruction the Illness Ideology and constructing an Ideology of Human Strengths and potential. In P. A. L. a. S. Joseph (Ed.), Positive Psychology In Practice. Hoboken, New Jersey: John Wiley \& Sons, Inc.

Mohammadi MR, Ghanizadeh H, Noorbala AA, Davidian H, Malek Afzali AA, \& HR, N. (2006). Prevalence of mood disorders in Iran. Iran J Psychiatry, 1, 59-64.

Noorbala A, Mohammadi K, \& SA, B. Y. (1987). A survey of psychi atric disorders in Tehran city. Hakim Magazine, 2(4), 212-223.

Rorschach, H. (1921). Psychodiagnostik; methodik und ergebnisse eines wahrnehmungsdiagnostischen experiments (deutenlassen von zufallsformen). Bern und Leipzig,: E. Bircher.

Ruini, C., \& Fava, G. A. (2009). Well-being therapy for generalized anxiety disorder. J Clin Psychol, 65(5), 510-519.

Ryff, C. D. (1989). Happiness is everything, or is it? Explorations on the meaning of psychological well-being. Personality and social psychology, 6, 1069-1081.

Ryff, C. D., \& Singer, B. (1996). Psychological well-being: meaning, measurement, and implications for psychotherapy research. Psychother Psychosom, 65(1), 14-23.

Seligman, M., Rashid, T., \& Parks, A. (2006). Positive psychotherapy. American Psychologist, (Nov)PP.774-788.

Sin, N. L., \& Lyubomirsky, S. (2009). Enhancing well-being and alleviating depressive symptoms with positive psychology interventions: a practice-friendly meta-analysis. Journal of Clinical of Psychology, 65(5), 467-487.

Vicente B, Kohn R, Rioseco P, Saldivia S, Baker C, \& S, T. (2004). Popula tion Prevalence of psychiatric disorders in chili: 6-month and I-month rates. BJ psychiatry, 184, 299-306.

Wood, A. M., Joseph, S., \& Maltby, J. (2009). Gratitude predicts psychologicalwell-being above the Big Five facets. Personality and Individual Differences 46, 443-447.

Zung, W. W. (1973). From art to science. The diagnosis and treatment of depression. Arch Gen Psychiatry, 29(3), 328-337.

Table1. Pre and post-scores on BDI II for CBT and WBT Groups

\begin{tabular}{c|c|c|c|c|c|c|c|c}
\hline BDI-II & \multicolumn{3}{|c|}{ Before therapy } & \multicolumn{2}{c|}{ After therapy } & \multicolumn{3}{c}{ Common } \\
\hline Variable & N & Mean & SD & Mean & SD & DF & F & Sig \\
CBT G & 20 & 33.00 & 3.35 & 12.35 & 4.31 & 3 & 3768.33 & .000 \\
WBT G & 20 & 45.35 & 5.30 & 8.05 & 1.82 & & & \\
\hline
\end{tabular}


Table2. Effect size for the scores of CBT and WBT groups

\begin{tabular}{c|cc}
\hline & CBT Group & WBT Group \\
\hline Cohen's $\boldsymbol{d}$ & 5.349794791354005 & 9.41331103708552 \\
Cohen's r (effect size) & 0.936684037967027 & 0.9781657927658106 \\
\hline
\end{tabular}

Table 3. F and p values for the sub-scale scores of BDI II for CBT and WBT groups

\begin{tabular}{|c|c|c|c|c|c|c|c|}
\hline \multirow{2}{*}{$\begin{array}{c}\text { Group } \\
\text { Variable }\end{array}$} & & \multicolumn{2}{|c|}{ Before Therapy } & \multicolumn{2}{|c|}{ After Therapy } & \multicolumn{2}{|c|}{ significance } \\
\hline & & Mean & SD & Mean & SD & $\mathbf{F}$ & $\mathbf{p}$ \\
\hline \multirow[t]{2}{*}{ Sadness } & CBT & 1.20 & .410 & .800 & .410 & & \\
\hline & WBT & 2.30 & .801 & .350 & .489 & 309.47 & .019 \\
\hline \multirow[t]{2}{*}{ Pessimism } & CBT & 1.90 & .552 & .850 & .366 & & \\
\hline & WBT & 1.65 & .489 & .450 & .510 & 574.82 & .003 \\
\hline \multirow[t]{2}{*}{ Past Failure } & CBT & 1.05 & .604 & .650 & .489 & & \\
\hline & WBT & 2.05 & .394 & .200 & .410 & 270.11 & .028 \\
\hline \multirow[t]{2}{*}{ Guilty Feelings } & CBT & 1.20 & .615 & .650 & .489 & & \\
\hline & WBT & 2.10 & .640 & .350 & .489 & 326.80 & .016 \\
\hline \multirow[t]{2}{*}{ Crying } & CBT & 1.00 & .561 & .750 & .444 & & \\
\hline & WBT & 2.10 & .911 & .300 & .470 & 229.23 & .023 \\
\hline \multirow[t]{2}{*}{ Agitation } & CBT & 1.00 & .648 & .750 & .444 & & \\
\hline & WBT & 2.10 & 1.29 & .450 & .510 & 133.32 & .038 \\
\hline \multirow[t]{2}{*}{ Loss of interest } & CBT & 1.05 & .686 & .650 & .489 & & \\
\hline & WBT & 2.10 & 1.16 & .250 & .444 & 169.60 & .043 \\
\hline \multirow[t]{2}{*}{ Indecisiveness } & CBT & 1.10 & .552 & .700 & .470 & & \\
\hline & WBT & 2.00 & .794 & .450 & .510 & 284.21 & .014 \\
\hline \multirow[t]{2}{*}{ Worthlessness } & CBT & 1.00 & .561 & .700 & .470 & & \\
\hline & WBT & 1.80 & .695 & .450 & .510 & 297.19 & .021 \\
\hline Loss of Interest in & CBT & 1.55 & .604 & .650 & .489 & & \\
\hline $\operatorname{sex}$ & WBT & 2.70 & .732 & .250 & .444 & 500.17 & .002 \\
\hline
\end{tabular}

Table 4

\begin{tabular}{cl|c|c}
\hline & Pearson Correlation & sum_post_pwb & sum_BDI-II_post \\
\hline sum_post_pwb & Pearson Correlation & 1 & $-.570(* *)$ \\
& Sig. (2-tailed) &. & .000 \\
& $\mathrm{~N}$ & 40 & 40 \\
sum_BDI-II_post & Pearson Correlation & $-.570(* *)$ & 1 \\
& Sig. (2-tailed) & .000 &. \\
& $\mathrm{~N}$ & 40 & 40 \\
\hline
\end{tabular}




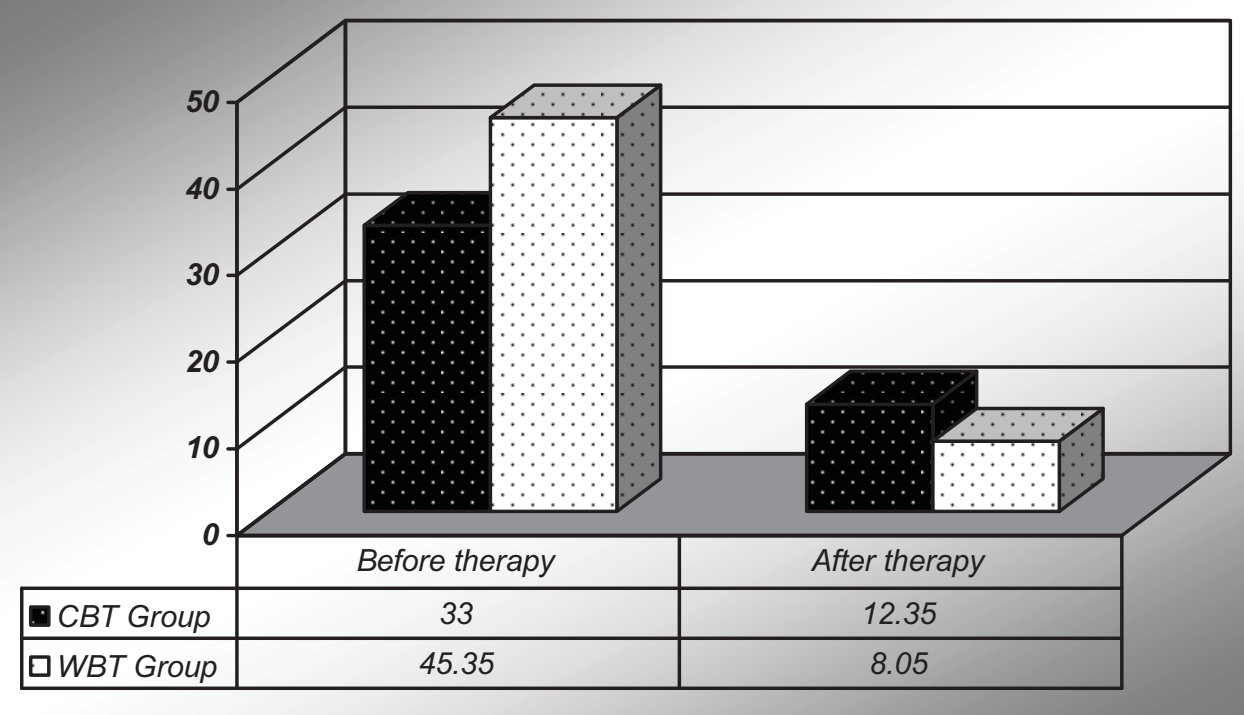

Figure 1.

For more clarity, Chart1 also illustrates the results

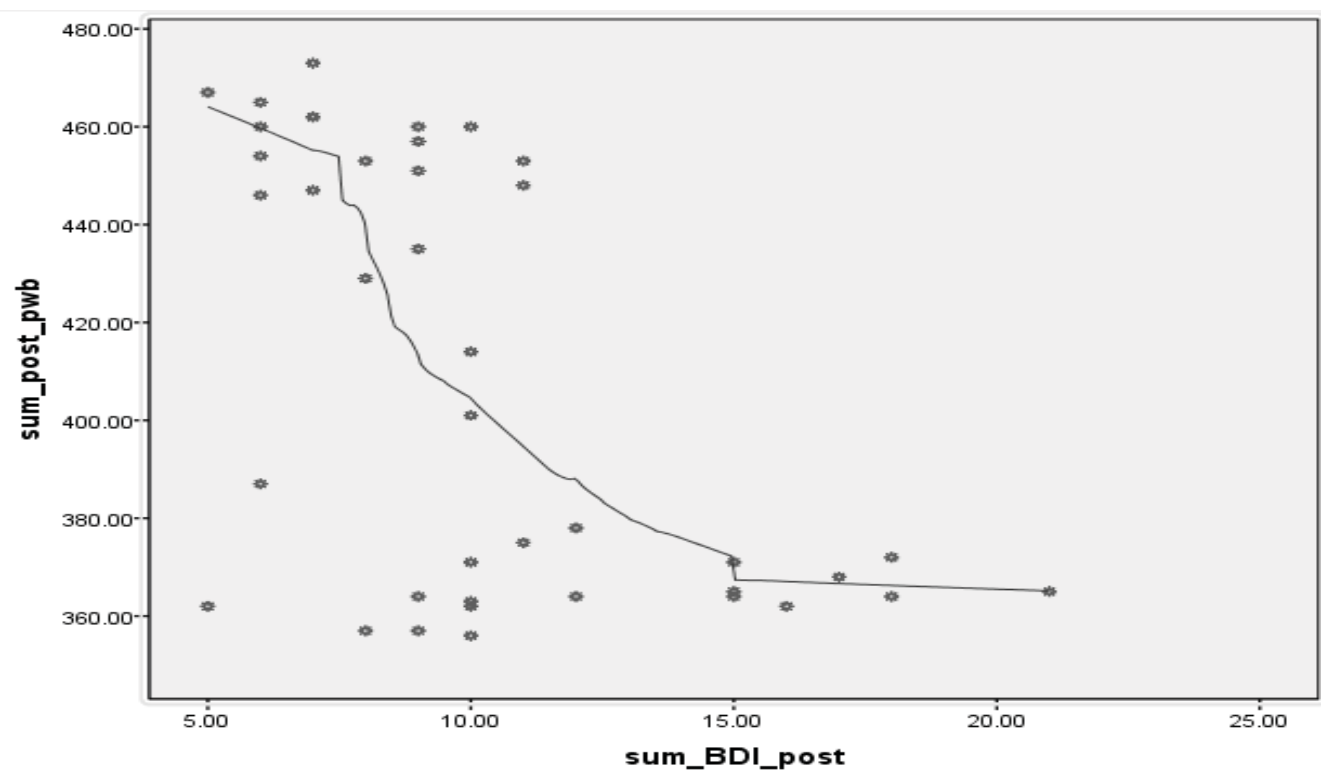

Figure 2. 